\title{
Antimicrobial agents of plant origin for the treatment of phlogistic-infectious diseases of the lower female genital tract
}

\author{
Francesco Gon', Fabrizia Gionechetti', Linda Furlanis', Lucia Corich', Cristina Lagatolla', \\ Cinzia Sanna², Mauro Ballero², Silvio Sosa', Roberto Della Loggia', Enrico Angelo Tonin' \\ I Dipartimento di Scienze della Vita, Università degli Studi di Trieste \\ 2 Dipartimento di Scienze Botaniche, Università degli Studi di Cagliari
}

Key Words: Antimicrobial agents, Natural products, Lower female genital tract

Proprietà antimicrobiche di prodotti di origine vegetale per il trattamento di patologie infettive del basso tratto genitale femminile

\section{SUMMARY}

The phlogistic-infectious diseases of the lower female genital tract are one of the most widespread obstetricgynecologic issues, due to treatment failures that cause frequent relapses and to the adverse effects of some commonly used drugs. The most common vaginal syndromes are due to uncontrolled growth of bacteria or fungi which replace the normal vaginal flora, causing phlogistic and infectious based diseases. These infections are treated with anti-inflammatory and antibiotic therapy; however, the emergence of resistant strains and the ability of many microorganisms to grow inside biofilms severely reduce the repertoire of useful agents. Thus, in the last years increasing interest has been focused toward compounds of plant origin with anti-microbial properties.

In the present work, we studied the antimicrobial activity of fractions obtained from endemic plants of Sardinia towards microorganisms that frequently are involved in vaginal infectious diseases: Streptococcus agalactiae, Gardnerella vaginalis and Candida albicans.

\section{INTRODUZIONE}

Le patologie flogistico-infettive del basso tratto genitale femminile rappresentano uno dei più diffusi problemi ostetrico-ginecologici, per le frequenti recidive e/o fallimenti terapeutici e per gli effetti avversi di alcuni dei farmaci impiegati (1). La sindrome vaginale più diffusa è dovuta a una crescita incontrollata di batteri o miceti, che spiazzano la normale flora vaginale e determinano l'insorgenza di patologie flogistico-infettive $(3,6)$. Queste infezioni sono affrontate con una terapia antibiotica e antinfiammatoria che, tuttavia, presenta una serie di problemi:

I) è sempre più frequente la comparsa di ceppi microbici resistenti ai farmaci utilizzati: questo rende difficoltoso il loro controllo (2);

II) molti microrganismi crescono all'interno di biofilm oltre che in forma planctonica: questo li rende più resistenti nei confronti di antibatterici, disinfettanti, $\mathrm{pH}$ estremi e difese dell'ospite (4).

Per questi motivi negli ultimi anni è aumentato l'interesse verso l'uso di agenti antimicrobici di origine vegetale, soprattutto per uso topico (5). L'applicazione topica è sicuramente vantaggiosa, poiché permette ai principi attivi di agire diretta- mente nella sede d'infezione, evitando la somministrazione sistemica e il conseguente metabolismo a livello gastrointestinale ed epatico.

In tal senso, è stata studiata l'attività antimicrobica di alcune frazioni ottenute da specie vegetali endemiche della Sardegna.

\section{MATERIALI E METODI}

Materiale vegetale ed estrazione.

Il seguente materiale vegetale $(500 \mathrm{~g})$ è stato raccolto nel 2010 e nel 2011 in diverse località della Sardegna: parti aeree di Vinca sardoa (Stearn) Pign., Apocynaceae (Iglesias); foglie di Arbutus unedo L., Ericaceae (Jerzu); foglie di Stachys glutinosa L., Lamiaceae (Jerzu); fiori di Asphodelus ramosus L., Liliaceae (Iglesias); parti aeree di Buplerum fruticosum L., Apiaceae (Osini); parti aeree di Glechoma sardoa Beg., Lamiaceae (Gennargentu). Mediante macerazione in etanolo ( $2.5 \mathrm{~L}$ x 3 volte) e rimozione del solvente, sono stati ottenuti i relativi estratti etanolici.

Ogni estratto è stato quindi disciolto in modo sequenziale con $n$-esano, acetato di etile e metanolo, ottenendo le relative frazioni esanica, etilacetica e metanolica.

Test di attività antimicrobica.

\section{Corresponding author: Cristina Lagatolla}

Dipartimento di Scienze della Vita - Università di Trieste

Via L. Giorgieri I - 34I27 Trieste, Italia - Tel. 0405588624 - Fax: 040558369 |

E-mail: clagatolla@units.it 
L'attività microbicida delle frazioni è stata valutata identificando la Minima Concentrazione Inibente (MIC) nei confronti dei ceppi di riferimento di tre microrganismi fra i più rilevanti nella patogenesi del basso tratto genitale femminile: Streptococcus agalactiae ATCC13813, Gardnerella vaginalis ATCC14018, Candida albicans ATCC90028.

Le MIC sono state valutate con il metodo della microdiluizione in brodo, utilizzando terreni e condizioni di incubazione riassunti in Tabella 1. Brevemente: $5 \times 10^{5}$ microrganismi sono stati inoculati in $200 \mu \mathrm{l}$ di terreno A addizionato con diluizioni seriali di estratto e incubati come indicato in Tabella 1.

La natura idrofobica dei campioni da saggiare rende difficoltosa la valutazione diretta del test: specialmente alle concentrazioni più elevate il contenuto del pozzetto appare torbido a prescindere dalla presenza o meno di crescita microbica, facendo così venir meno il principio visivo del test. Per ovviare al problema, $5 \mu \mathrm{l}$ di brodocoltura sono stati ulteriormente seminati su terreno solido (B), in modo da valutare la presenza o meno di batteri vivi.

\section{RISULTATI}

I risultati preliminari indicano livelli di suscettibilità molto diversi da parte dei tre microrganismi nei confronti delle frazioni studiate. G. vaginalis ha mostrato una buona suscettibilità a diverse frazioni, con MIC spesso < $200 \mu \mathrm{g} / \mathrm{ml}$ (Tabella 2).

Nei casi in cui erano disponibili campioni raccolti in due annate diverse, alcune frazioni hanno presentato un differente livello di attività, probabilmente dovuto a differenze nei costituenti chimici del materiale vegetale tra i due anni di raccolta.

S. agalactiae è risultato suscettibile solo a concentrazioni elevate (dell'ordine di $\mathrm{mg} / \mathrm{ml}$ ) di frazioni di Stachys glutinosa (Tabella 3), mentre ha mostrato MIC $>10 \mathrm{mg} / \mathrm{ml}$ (la più alta concentrazione saggiata) per tutte le altre frazioni.

C. albicans è risultata resistente a quasi tutte le frazioni saggiate. Solo la frazione esanica di $G$. sardoa presentava una blanda attività antifungina, con una MIC pari a $5 \mathrm{mg} / \mathrm{ml}$.

\section{CONCLUSIONI}

Vista la complessità di gestione con farmaci convenzionali delle patologie flogistico-infettive del basso tratto genitale femminile, è interessante aver riscontrato attività antimicrobica in alcune delle frazioni saggiate.

I risultati ottenuti hanno comunque carattere preliminare e dovranno essere confermati, verificando con particolare attenzione le differenze riscontrate tra frazioni raccolte in annate diverse.

In una fase successiva, lo studio sarà rivolto a identificare i principi responsabili dell'attività microbicida all'interno delle diverse frazioni.

Tabella 2. MIC nei confronti di G. vaginalis $(\mu \mathrm{g} / \mathrm{ml})$.

\begin{tabular}{llcc}
\hline SPECIE & FRAZIONE & \multicolumn{2}{c}{ ANNO DI } \\
VEGETALE & & \multicolumn{2}{c}{ RACCOLTA } \\
\hline Vinca sardoa & & 20 I I \\
\cline { 2 - 4 } & Esanica & 78 & 3 I 2 \\
\cline { 2 - 4 } & Etilacetica & 2500 & 2500 \\
\hline Arbutus unedo & Esanica & 39 & 78 \\
\cline { 2 - 4 } & Etilacetica & 39 & 39 \\
\cline { 2 - 4 } & Metanolica & 312 & 2500 \\
\hline Stachys glutinosa & Esanica & 19.5 & 19.5 \\
\cline { 2 - 4 } & Etilacetica & 19.5 & 4.9 \\
\cline { 2 - 4 } & Metanolica & 19.5 & 19.5 \\
\hline Asphodelus ramosus & Esanica & 19.5 & $/$ \\
\cline { 2 - 4 } & Etilacetica & 39 & $/$ \\
\cline { 2 - 4 } & Metanolica & 39 & $/$ \\
\hline Buplerum fruticosum & Esanica & 19.5 & $/$ \\
\cline { 2 - 4 } & Etilacetica & 312 & $/$ \\
\cline { 2 - 4 } & Metanolica & 625 & $/$ \\
\hline Glechoma sardoa & Esanica & $/$ & 2.4 \\
\cline { 2 - 4 } & Etilacetica & $/$ & 312 \\
\cline { 2 - 4 } & Metanolica & $/$ & 1250 \\
\hline
\end{tabular}

Tabella 3. MIC nei confronti di S. agalactiae $(\mathrm{mg} / \mathrm{ml})$.

\begin{tabular}{llcc}
\hline $\begin{array}{l}\text { SPECIE } \\
\text { VEGETALE }\end{array}$ & FRAZIONE & \multicolumn{2}{c}{$\begin{array}{l}\text { ANNO DI } \\
\text { RACCOLTA }\end{array}$} \\
\hline & & $\mathbf{2 0 I 0}$ & $\mathbf{2 0 ~ I ~ I ~}$ \\
\hline tachys glutinosa & & 2.5 & 2.5 \\
\cline { 2 - 4 } & Esanica & 5 & 1.25 \\
\cline { 2 - 4 } & Etilacetica & 1.25 & $/$ \\
\cline { 2 - 4 }
\end{tabular}

Tabella I. Terreni e condizioni di crescita utilizzati per la valutazione delle MIC.

\begin{tabular}{|c|c|c|c|}
\hline Microrganismo & $\begin{array}{c}\text { Terreno A } \\
\text { (microdiluizione) }\end{array}$ & Condizioni di incubazione & $\begin{array}{c}\text { Terreno } \mathrm{B} \\
\text { (conferma attività microbicida) }\end{array}$ \\
\hline S. agalactiae & $\mathrm{BHI}$ broth & $24 \mathrm{~h}$ a $37^{\circ} \mathrm{C}$ & $\mathrm{BHI}$ agar \\
\hline G. vaginalis & Brucella broth $^{a}$ & $48 \mathrm{~h}$ a $37^{\circ} \mathrm{C}$ in $6 \% \mathrm{CO}_{2}$ & Columbia agar sangue \\
\hline C. albicans & RPMI broth & $24 \mathrm{~h}$ a $30^{\circ} \mathrm{C}$ & Sabouraud agar \\
\hline
\end{tabular}

$\mathrm{a}$ :alcuni estratti presentano attività emolitica: questo rende inutilizzabili i terreni addizionati con sangue. 


\section{BIBLIOGRAFIA}

1. Burke et al. In "G\&G The pharmacological basis of therapeutics-eleventh edition". McGraw-Hill, NY, 2005; 671-715.

2. Cernicka J, Subik J. Resistance mechanisms in fluconazole-resistant Candida albicans isolates from vaginal candidiasis. Int J Antimicrob Agents. 2006; 27 : 403-8.

3. Mårdh PA. Bacterial vaginosis: a threat to reproductive health? Historical perspectives, current knowledge, controversies and research demands. Eur J Contracept Reprod Health Care. Sep 2000; 5 (3): 208-19.

4. Patterson JL, Girerd PH, Karjane NW, Jefferson KK. Effect of biofilm phenotype on resistance of Gardnerella vaginalis to hydrogen peroxide and lactic acid. Am J Obstet Gynecol 2007; 197: 170-7.

5. Potterat O, Hamburger M. Drug discovery and development with plant-derived compounds. Progr Drug Res 2008; 65: 47-118.

6. Sobel JD. Vulvovaginal candidosis. Lancet 2007; 369: 1961-71. 